7th International Symposium on Superalloy 718 and Derivatives Edited by: E.A. Ott, J.R. Groh, A. Banik, I. Dempster, T.P. Gabb, R. Helmink, X. Liu, A. Mitchell, G.P. Sjöberg, and A. Wusatowska-Sarnek TMS (The Minerals, Metals \& Materials Society), 2010

\title{
EVOLUTION OF $\delta$ PHASE MICROSTRUCTURE IN ALLOY 718
}

\author{
Sundararaman Mahadevan ${ }^{1}$, Sachin Nalawade ${ }^{1}$, Jung Bahadur Singh ${ }^{1}$, Amit Verma ${ }^{1}$, \\ Bhaskar Paul ${ }^{2}$ and Kishore Ramaswamy ${ }^{1}$ \\ ${ }^{1}$ Structural Metallurgy Section, Materials Group, BARC, Mumbai 4000 85, India; \\ ${ }^{2}$ Refractory Metals Development Section, Materials Group, BARC, Mumbai 4000 85, India
}

Keywords: Alloy $718, \delta$ phase, $\mathrm{Ni}_{3} \mathrm{Nb}$ phase, microstructure evolution

\begin{abstract}
Though Alloy 718 is strengthened by $\gamma^{\prime \prime}$ precipitation, the nucleation of $\delta$ phase is used to stabilize grain size and also to improve its high temperature stress rupture properties. Large body of work has been reported in the literature on the precipitation of the $\gamma^{\prime \prime}$ phase. However, hardly very few reports are available on the evolution of $\delta$ particles in Alloy 718. The present paper reports the results of a detailed microstructural investigation carried out using Transmission Electron Microscopy (TEM) and Scanning Electron Microscopy (SEM) on the evolution of $\delta$ microstructure. Based on the SEM observations, three different mechanisms have been proposed for the generation of intersecting $\delta$ microstructure. In addition to the stacking faults in the matrix and $\gamma^{\prime \prime}$ particles acting as nuclei for $\delta$ phase formation, a new mechanism has been observed where the faults generated on the conjugate habit plane by dissociation of misfit dislocation on the interface of a $\delta$ variant acted as nuclei for another $\delta$ variant precipitation.
\end{abstract}

\section{Introduction}

Alloy 718 finds several applications in aerospace, petrochemical and nuclear industries due to its excellent high temperature strength and good oxidation and corrosion resistance up to very high temperatures [1]. The major phases that precipitate in a face centered cubic (fcc) matrix are metastable $\gamma^{\prime \prime}$ (tetragonal $\mathrm{D}_{22}$ structure), equilibrium $\gamma^{\prime}$ (cubic $\mathrm{L}_{2}$ structure) and equilibrium $\delta$ (orthorhombic $\mathrm{D}_{\mathrm{a}}$ structure) phases [2-6]. The age hardening in this alloy is mainly brought about by the precipitation of the $\gamma^{\prime \prime}$ phase particles that have coherent disc shaped morplology with $\{100\}$ habit plane. Some strengthening is brought about by the precipitation of coherent $\gamma^{\prime}$ particles as well [4]. The equilibrium phase corresponding to the metastable $\gamma^{\prime \prime}$ phase is the $\delta$ phase [7]. Alloy 718 and other $\gamma^{\prime \prime}$ phase strengthened nickel and nickel-iron base superalloys, are susceptible to the $\delta$ phase formation that can evolve during processing or during service [8]. On prolonged exposure at intermediate temperatures, the $\delta$ phase forms as a transformation product of $\gamma^{\prime \prime}$ phase [9-11]. At relatively lower temperatures, $\delta$ phase nucleates at austenite grain boundaries as well as coherent and incoherent twin boundaries, while at higher temperatures they precipitate intragranularly [12]. Though the $\delta$ precipitates are incoherent and their precipitation is known to reduce the strength of $\gamma^{\prime \prime}$ strengthened alloys, controlled precipitation of $\delta$ phase has some beneficial effects like grain stabilization and good stress rupture properties [13]. 
The knowledge gained on the precipitation of the metastable and the equilibrium phases $\left(\gamma^{\prime \prime}\right.$ and $\delta$ ) has been skillfully adopted by industry to decide the standard heat treatment procedure to produce this alloy in the age hardenable condition [1-8]. The procedure involves solution treatment at $954{ }^{\circ} \mathrm{C}$ followed by ageing at $720{ }^{\circ} \mathrm{C}$ and $620{ }^{\circ} \mathrm{C}$ for specific periods of time. Heat treatment at former temperature produces blocky $\delta$ particles, mainly at grain boundaries, to stabilize the grains, while heat treatments at latter temperatures are given to precipitate $\gamma^{\prime \prime}$ particles in the matrix to strengthen the alloy. Heat treatments at temperatures lower than $954{ }^{\circ} \mathrm{C}$ has been employed to generate a microstructure (consisting of high volume fraction of uniformly dispersed fine $\delta$ particles) that is used during forging operations to produce fine grain grades of Alloy $718[14,15]$. Similarly, any abnormal rise in temperature or stress during service can also result in complete conversion of $\gamma^{\prime \prime}$ precipitates to $\delta$ particles $[10,16]$. A considerable amount of work has been reported on the nucleation and growth of the $\delta$ phase $[9$, $12,17-19]$. However, hardly any information is available in the literature on the evolution of $\delta$ microstructures with high volume fraction. At these high temperatures, the $\delta$ particles have been reported to have needle, plate as well as globular morphologies $[9,20]$. The main objective of the present study is to look into some of the issues pertaining to morphology and evolution of $\delta$ phase. The present paper reports the results of a detailed microstructural investigation carried out on these aspects in specimens heat treated at $900{ }^{\circ} \mathrm{C}$, using TEM and SEM. The evolution of microstructure consisting of intersecting $\delta$ particles is explained on the basis of various mechanisms put forward in the literature as well as a new one proposed in this paper.

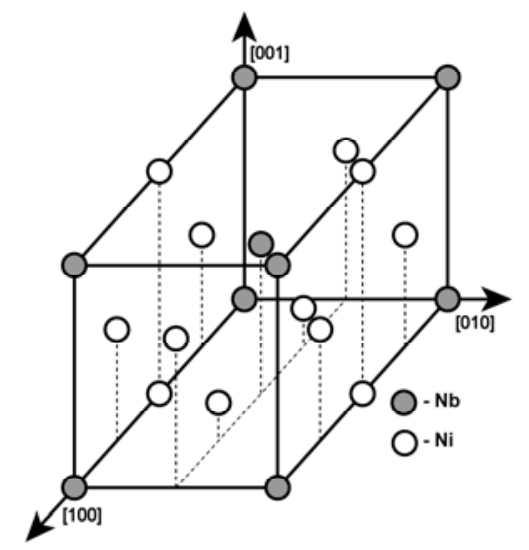

Figure 1. The unit cell of $\mathrm{Ni}_{3} \mathrm{Nb}$ $\mathrm{D} 0_{\mathrm{a}}$ structure of $\delta$ phase.

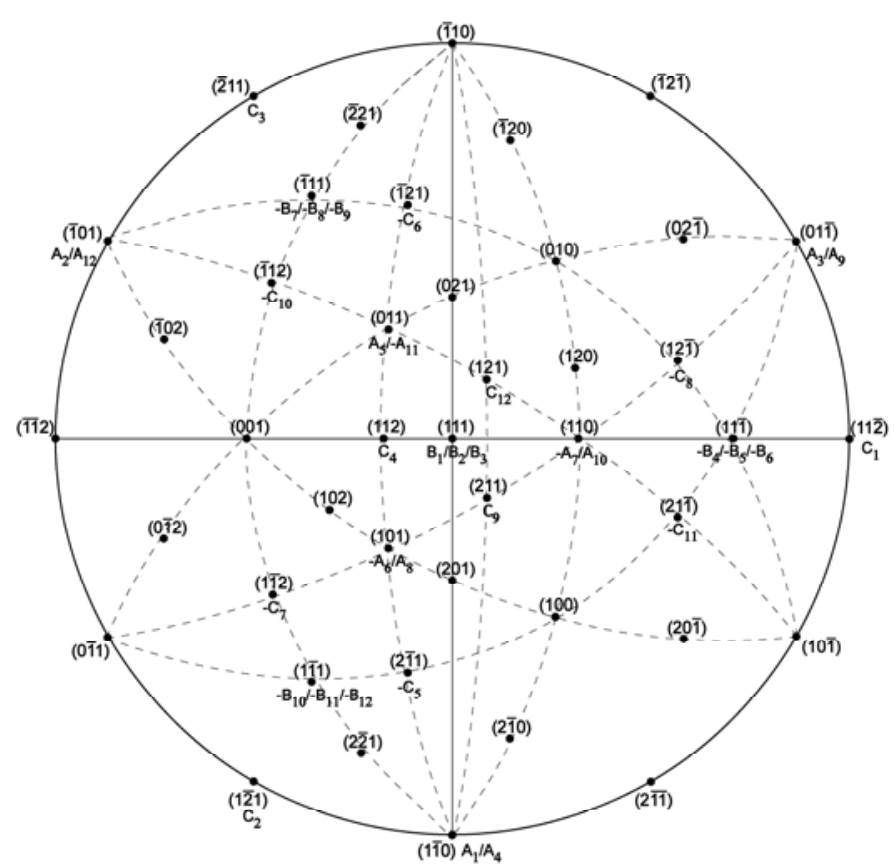

Figure 2. Orientation relation of 12 variants of $\delta$ phase on (111) cubic stereogram. 

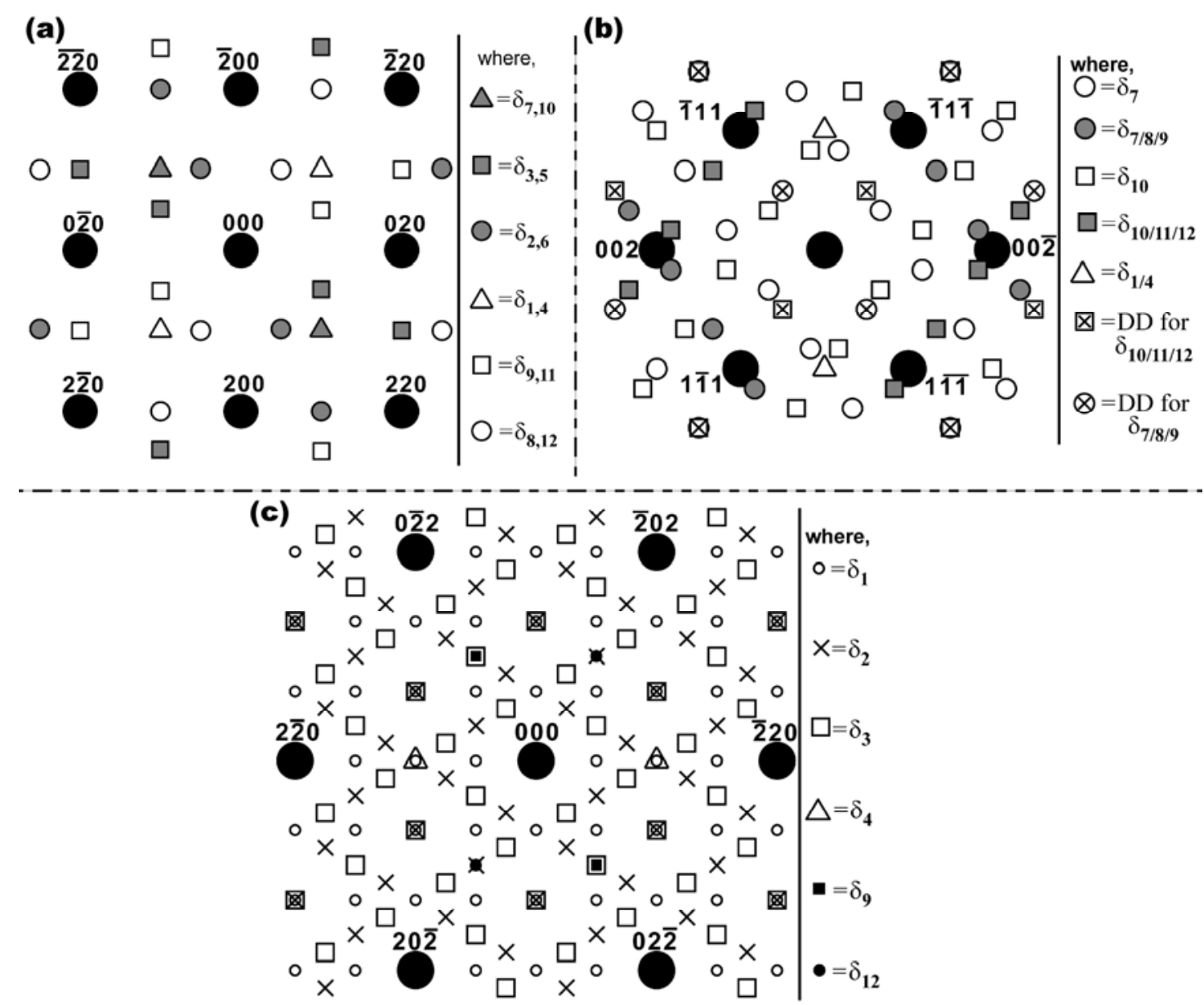

Figure 3. Simulated electron diffraction patterns for (a) [001], (b) [110] and (c) [111] beam directions depicting the positions of superlattice reflections corresponding to different variants of the $\delta$ phase. Some of the superlattice reflections correspond to more than one variant. The variants each superlattice symbol represents are explained adjacent to each pattern.

\section{Crystallographic Aspects of $\delta$ Phase}

The $\delta$ phase has an orthorhombic $\mathrm{D}_{0}$ a structure with $\mathrm{Ni}_{3} \mathrm{Nb}$ stoichiometry $[9,21]$ and its unit cell is illustrated in Figure 1. The orientation relation between the $\mathrm{D} 0_{\mathrm{a}}$ structure and fcc matrix as reported in the literature can be expressed as $\{111\}_{\text {fcc }} / /(010)_{\mathrm{D}_{\mathrm{a}}}$ and $<1 \overline{1} 0>_{\mathrm{fcc}} / /[100]_{\mathrm{D}_{\mathrm{a}}}$. It is clear from this relationship that on each close packed plane, three variants of the $\delta$ phase will form with their $\mathbf{A}$ axes along any one of the close packed direction of the fcc lattice. Thus twelve variants of the $\delta$ phase with four distinct habit planes will form in the fcc matrix. Nomenclature of these variants and their lattice correspondence is given in Table 1. The orientation relationship of these variants with fec matrix are shown on a standard [111] cubic stereographic projection (Figure 2). In this stereogram, $A_{i}, B_{i}$ and $C_{i}$ denote [100], [010] and [001] directions of the $\delta$ unit cell, while the subscript ' $i$ ' corresponds to variant number. Using 
the correspondence relationship between the unit cell of fcc and each variant of $\delta$ particle [22], electron diffraction patterns were simulated for each variant for different beam directions in the matrix. Such patterns for [001], [110] and [111] beam directions are shown in Figure 3 . Different variants could be identified from the presence of superlattice spots at specific positions in the diffraction pattern. These patterns are compared with the experimentally obtained diffraction patterns to uniquely identify different variants of the $\delta$ phase.

\section{Experimental}

The nominal chemical composition of Alloy 718 used in this study is given in Table 2. Flat coupons were prepared by cold rolling of the as received alloy. Samples, sealed in quartz tube under helium atmosphere, were solution annealed (ST) at $1100{ }^{\circ} \mathrm{C}$ for $1 \mathrm{~h}$ to dissolve all intermetallic phases. Some of these ST samples were heat treated at $900{ }^{\circ} \mathrm{C}$ for $100 \mathrm{~h}$ to precipitate maximum volume fraction of $\delta$ phase (henceforth designated as DELTA specimens). Some of these ST samples were heat treated at $900{ }^{\circ} \mathrm{C}$ for $100 \mathrm{~h}$ to precipitate maximum volume fraction of $\delta$ phase (henceforth designated as DELTA specimens). The microstructure of ST and

Table 1. Lattice correspondence and variant nomenclature of the $\delta$ phase in relation to the fcc lattice.

\begin{tabular}{|c|c|c|c|}
\hline Variant & {$[100]_{\mathrm{DO}_{\mathrm{a}}}$} & {$[010]_{\mathrm{DO}_{\mathrm{a}}}$} & {$[001]_{\mathrm{DO}_{\mathrm{a}}}$} \\
\hline$\delta_{1}$ & {$[1 \overline{1} 0]$} & $\frac{2}{3}[111]$ & $\frac{1}{2}[11 \overline{2}]$ \\
\hline$\delta_{2}$ & {$[\overline{1} 01]$} & $\frac{2}{3}[111]$ & $\frac{1}{2}[1 \overline{2} 1]$ \\
\hline$\delta_{3}$ & {$[01 \overline{1}]$} & $\frac{2}{3}[111]$ & $\frac{1}{2}[\overline{2} 11]$ \\
\hline$\delta_{4}$ & {$[1 \overline{1} 0]$} & $\frac{2}{3}[\overline{1} \overline{1} 1]$ & $\frac{1}{2}[112]$ \\
\hline$\delta_{5}$ & [011] & $\frac{2}{3}[\overline{1} \overline{1} 1]$ & $\frac{1}{2}[\overline{2} 1 \overline{1}]$ \\
\hline$\delta_{6}$ & {$[\overline{1} 0 \overline{1}]$} & $\frac{2}{3}[\overline{1} \overline{1} 1]$ & $\frac{1}{2}[1 \overline{2} \overline{1}]$ \\
\hline$\delta_{7}$ & {$[\overline{1} \overline{1} 0]$} & $\frac{2}{3}\left[\begin{array}{lll}1 & \overline{1} & 1\end{array}\right]$ & $\frac{1}{2}[\overline{1} 1 \overline{2}]$ \\
\hline$\delta_{8}$ & [101] & $\frac{2}{3}\left[\begin{array}{lll}1 & \overline{1} & 1\end{array}\right]$ & $\frac{1}{2}[\overline{12} 1]$ \\
\hline$\delta_{9}$ & {$[01 \overline{1}]$} & $\frac{2}{3}\left[\begin{array}{lll}1 & \overline{1} & \overline{1}\end{array}\right]$ & $\frac{1}{2}[211]$ \\
\hline$\delta_{10}$ & [011] & $\frac{2}{3}[\overline{1} 1 \overline{1}]$ & $\frac{1}{2}[1 \overline{12}]$ \\
\hline$\delta_{11}$ & {$\left[\begin{array}{ll}0 & \overline{1} \\
1\end{array}\right]$} & $\frac{2}{3}[\overline{1} 1 \overline{1}]$ & $\frac{1}{2}[\overline{2} \overline{1} 1]$ \\
\hline$\delta_{12}$ & ['̄01] & $\frac{2}{3}[\overline{1} 1 \overline{1}]$ & $\frac{1}{2}[121]$ \\
\hline
\end{tabular}


DELTA specimens were examined in optical, scanning and transmission electron microscopes. Optical and SEM specimens were prepared by electrolytic etching (in a saturated solution of oxalic acid at 10 volts dc for about 10 to 20 seconds) of specimens polished to a mirror finish by grinding on successive grades of silicon carbide paper and diamond paste. TEM specimens were prepared from jet thinning of $3 \mathrm{~mm}$ discs punched out from thin foils in a dual jet electropolishing unit using an electrolytic bath containing ethanol and perchloric acid mixture in the ratio of $80: 20$ maintained at $-30{ }^{\circ} \mathrm{C}$ temperature.

Table 2. Chemical composition of Alloy 718

\begin{tabular}{|c|c|c|c|c|c|c|c|c|c|c|}
\hline Element & $\mathrm{Ni}$ & $\mathrm{Cr}$ & $\mathrm{Fe}$ & $\mathrm{Nb}$ & $\mathrm{Mo}$ & $\mathrm{Al}$ & $\mathrm{Ti}$ & $\mathrm{Mn}$ & $\mathrm{Si}$ & $\mathrm{C}$ \\
\hline Con. (wt.\%) & 52.69 & 18.37 & 18.06 & 6.00 & 2.91 & 1.00 & 0.45 & 0.21 & 0.29 & 0.02 \\
\hline
\end{tabular}

\section{Results}

\section{Microstructure of ST And DELTA Samples}

Optical micrograph of ST specimens (Figure 4a) revealed equi-axed grains of nearly uniform size $(\sim 60 \mu \mathrm{m})$. TEM investigations revealed a single phase austenite microstructure with low density of dislocations generated probably to accommodate thermal stresses and they are predominantly arranged in planar arrays (Figure $4 \mathrm{~b}$ ). Absence of any superlattice reflections in $<001>$ selected area diffraction (SAD) pattern (inset in Figure $4 \mathrm{~b}$ ) indicated that the specimen was free of any intermetallic precipitates and the matrix is in single phase. Ageing of the specimen has resulted in profuse precipitation of uniformly distributed $\delta$ particles in the matrix as revealed in Figure 5. The particles appeared to exhibit a morphology that looked like needle in the viewing direction in optical, TEM and SEM examination (Figure 5) and were lying along specific crystallographic directions in the matrix. TEM investigations revealed that the interfaces of particles were aligned along the trace of $\{111\}$ planes. Some blocky precipitates could be seen at grain boundaries as well as within the matrix (Figure 5b). In fact, the tiny dot

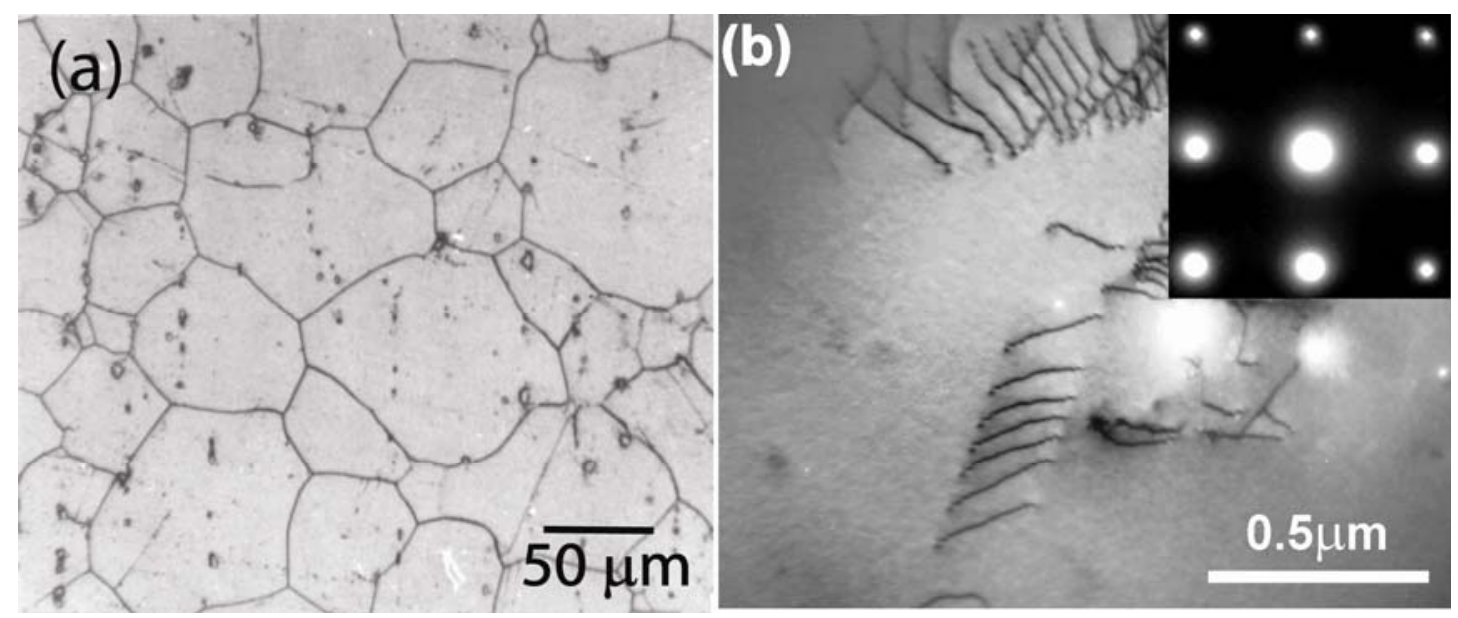

Figure 4. (a) Optical and (b) BF micrographs of ST specimen; Inset in (b) is $<001>$ SAD pattern from corresponding areas. 
like features seen in Figure 5b would correspond to cut sections of globular precipitates or needle shaped precipitates lying perpendicular to polished surface. If the particles have true needle shaped morphology, then their tip should normally appear as more or less point shaped. Finite width at the tip of many $\delta$ particles (marked by arrows in Figure 5c) indicated that their morphology is not needle shaped. Identification of true morphology of the particles is described

Table 3. Composition of the $\delta$ phase and austenite matrix in DELTA and ST specimens determined using EDS

\begin{tabular}{|c|c:c:c:c:c:c:c|}
\hline \multirow{2}{*}{ Phase } & \multicolumn{6}{|c|}{ Atomic Concentration of elements (in at \%) } \\
\cline { 2 - 7 } & $\mathrm{Fe}$ & $\mathrm{Cr}$ & $\mathrm{Nb}$ & $\mathrm{Ti}$ & $\mathrm{Al}$ & $\mathrm{Mo}$ & $\mathrm{Ni}$ \\
\hline$\delta$ & 11.65 & 12.73 & 12.52 & 2.35 & - & 2.87 & Bal. \\
\hline DELTA matrix & 20.46 & 22.34 & 2.41 & 0.95 & 2.39 & 2.53 & Bal. \\
\hline ST matrix & 18.6 & 20.36 & 3.75 & 1.32 & 2.09 & 2.36 & Bal. \\
\hline
\end{tabular}

in the next section. The volume fraction of the $\delta$ particles was theoretically determined from the knowledge of $\delta$ forming solute (niobium) in the matrix of ST and DELTA (Table 3) specimens, in the precipitate, as well as the molar volume of $\delta$ phase using the relationship given in reference [22] and was found to be $\sim 11.5 \%$. This value is in good agreement with the maximum equilibrium volume fraction of $\delta$ phase $(\sim 12 \%)$ theoretically estimated by Desvallées et al. [14].

\section{Morphology of $\delta$ Precipitates}

In order to determine the true morphology of $\delta$ particles, a specimen was deeply etched so as to preferentially remove the matrix and leave behind a network of only precipitates on the surface. The $\delta$ precipitates in the specimen now exhibited a ribbon or plate like morphology (Figure 6).
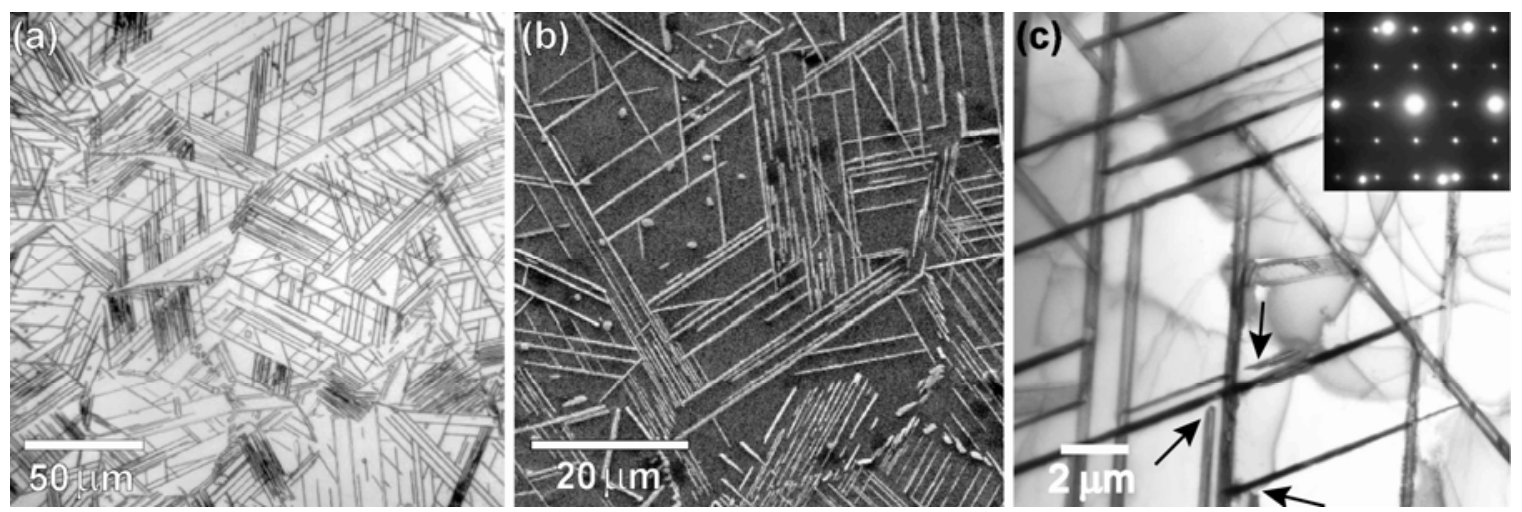

Figure 5. (a) Optical, (b) SEM and (c) BF TEM micrograph of DELTA specimen. Inset in (c) is $<110>$ SAD pattern. 
Two variants appeared to be lying inclined to the sample surface while the other two variants lay normal to it. Some of the salient observations of this study, depicted in Figure 7, are as follows:

1) The $\delta$ precipitates have plate morphology (Figure 7a).

2) The $\delta$ plates of one orientation often appeared to pass/pierce through another variant (marked by arrows in Figure 7d).

3) In some regions, one variant appeared to have enveloped another variant probably during its growth (see double arrow marks in Figure 7c to 7f).

4) In some other areas, one variant is lying on top of another, with part of it partially embedded in the second phase (see region shown within box in Figure $7 \mathrm{~b}$ ).

These observations indicated that the $\delta$ precipitates have plate shaped morphology and their intersecting network had formed as a result of growth.

\section{$\underline{\text { Intersection of } \delta \text { Precipitates }}$}

With a view to get an insight into the nature of the intersection of $\delta$ particles, a detailed transmission electron microscopy investigation was carried out. The major difficulty encountered in the examination of DELTA specimen in TEM was the presence of large number of bend contours in the electron transparent region of the thin foil. This hampered the tilting of area of interest from one zone to another to get the requisite information. It is known from earlier investigations [5] that the nucleation of fine precipitates in the matrix considerably reduced the formation of bend contours in electron transparent regions of TEM specimens. Therefore, an ageing treatment at $725^{\circ} \mathrm{C}$ for $50 \mathrm{~h}$ was given to DELTA specimens. This

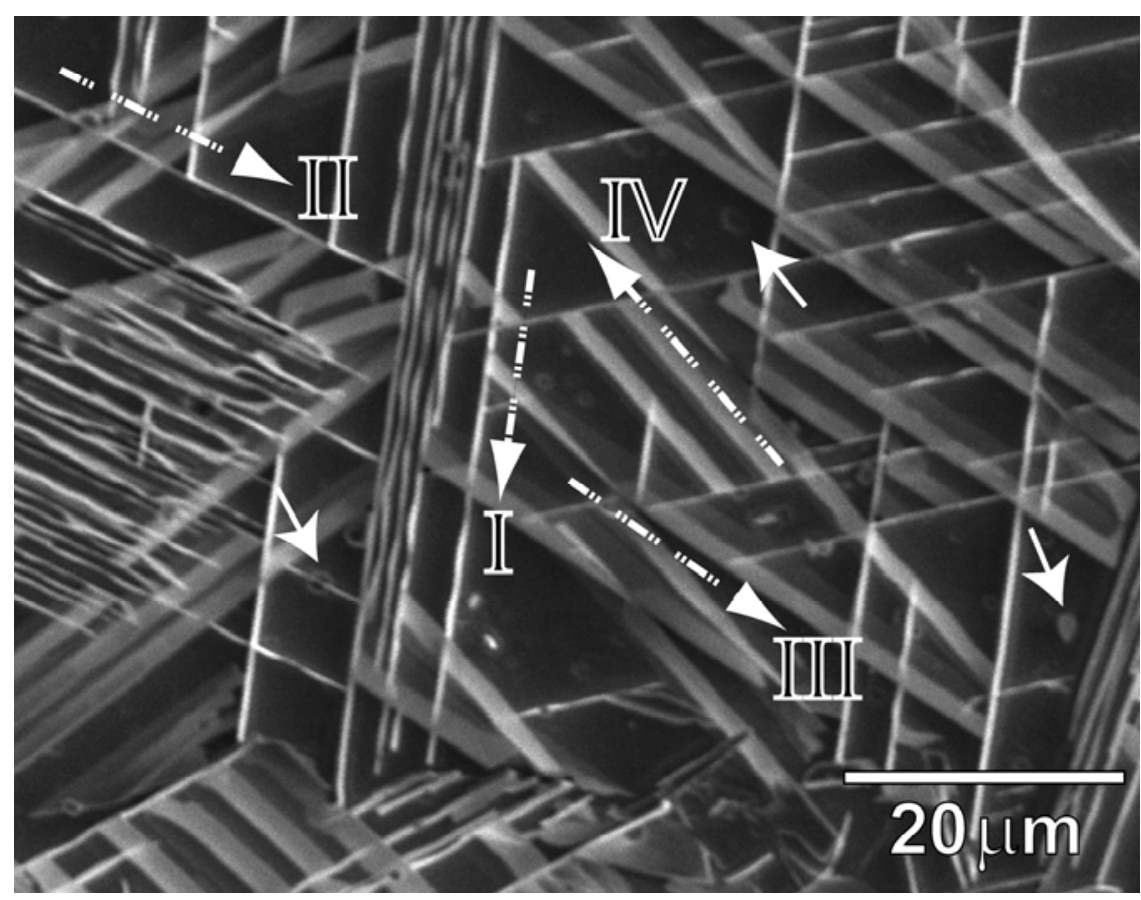

Figure 6. SEM micrograph of slightly over etched DELTA specimen showing intersecting $\delta$ precipitates arranged in four different orientations (marked by big arrows). The presence of small spherical or circular precipitates is shown by small arrows 
treatment resulted in the precipitation of small amount of niobium solute, in excess of the equilibrium solubility limit in the matrix at this temperature, as very fine $\gamma^{\prime \prime}$ particles uniformly distributed between $\delta$ particles (Figure 8) and no bend contours were seen in the electron transparent area. This treatment was not expected to result in the formation of new $\delta$ precipitates nor increase in its volume fraction $[5,9]$. Hence, it was assumed that this heat treatment did not alter any of the features of $\delta$ precipitation formed during DELTA treatment. Intersection of two variants of $\delta$ (marked A and B) could be seen in Figure 8. The junctions where these variants intersected are numbered 1 to 5 . At junction 1, 2 and 4, variant $B$ was continuous (as inferred from its width and contrast) while A variant was continuous across junction 3 and 5. Slight constriction could be noticed at the intersections. This indicated that many of these intersections
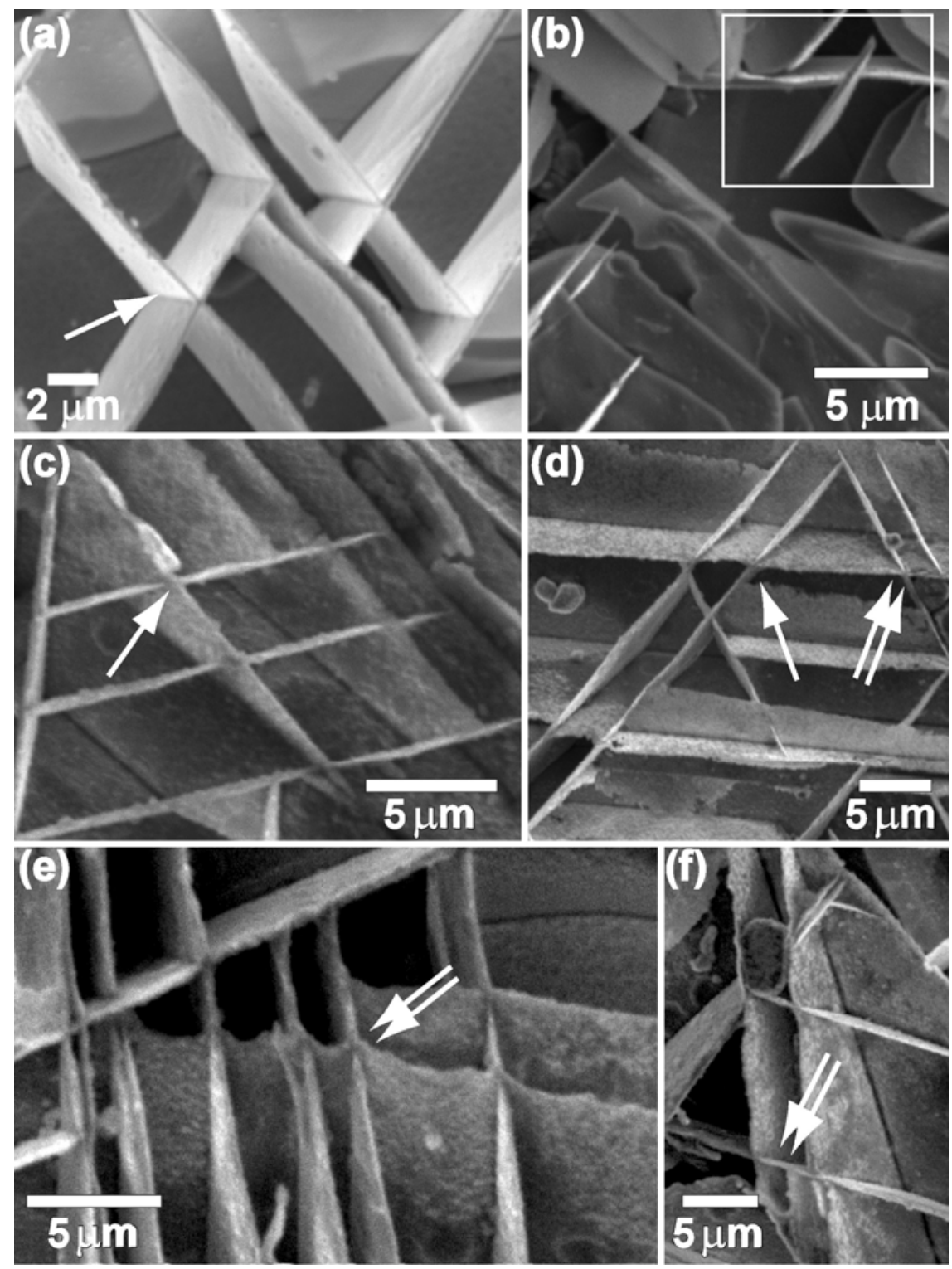

Figure 7. SEM micrographs of deep etched DELTA specimen revealing plate morphology and intersecting nature of $\delta$ precipitates. 
may have been generated by shearing of one variant by the growth/propagation of another variant. One such junction is depicted in Figure 9. The orientation of the beam was along [110] direction and the SAD pattern obtained from the junction is shown in Figure 10. This pattern was compared with the simulated diffraction patterns [22] to identify the variants and the keys corresponding to them are shown in Figures $10 \mathrm{~b}$ and c. This analysis indicated that the $\delta$ plates corresponded to variants $\delta_{7}$ and $\delta_{10}$ as per the nomenclature given in Table 1 and the habit planes of both variants are contained in the beam direction. The $\delta$ plate (marked B) that was continuous across the junction had sheared the $\delta$ plate (marked A) that was discontinuous. The relative shift of variant $A$ across the intersection also confirmed this point. The displacement of the upper half of the $\delta$ plate A with respect to the lower half was measured to be $\sim 33.2 \mathrm{~nm}$. Assuming this shift was the result of shear along close packed direction in $\{111\}$ planes, the magnitude of the displacement was estimated from the width of $\delta$ plate B $(102.5 \mathrm{~nm})$ to be $\sim 35.6 \mathrm{~nm}$. The reasonable agreement between the experimentally determined value and the estimated one also suggested that the intersection of $\delta$ variants is occurring by one variant shearing another.

Another interesting feature of this study was the observation of nucleation of $\delta$ variant with its habit plane different from the variant on which it had formed (marked by dotted circles in Figure 8 ). The ramification of this observation on growth and intersection of $\delta$ variants is discussed in the next section.

\section{Discussion}

The results presented in the last section clearly established that profuse precipitation of $\delta$ particles had occurred during aging at $900{ }^{\circ} \mathrm{C}$ for $100 \mathrm{~h}$. In the literature, $\delta$ particles have been reported to have either globular, needle or plate like morphology $[9,14,19]$. From the optical, SEM and TEM observations in Figure 5, it appeared that the $\delta$ particles have needle and blocky

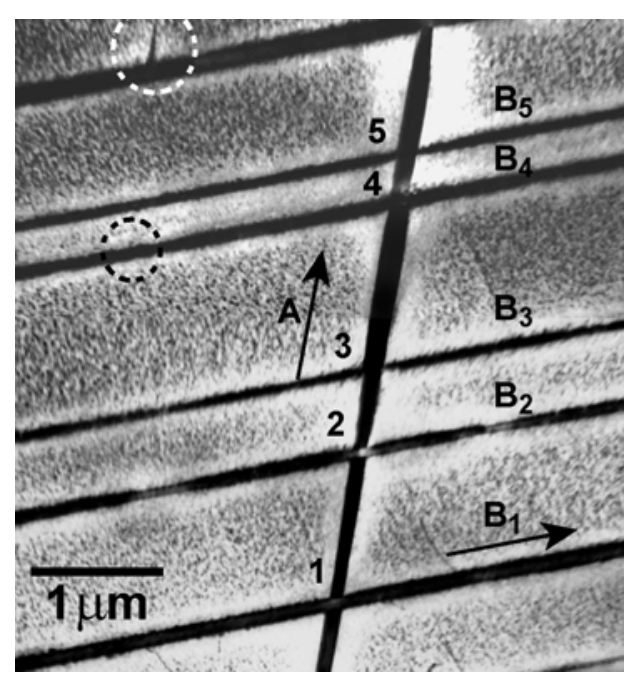

Figure 8. BF micrograph of DELTA specimen showing intersecting $\delta$ precipitates with two different habit planes.

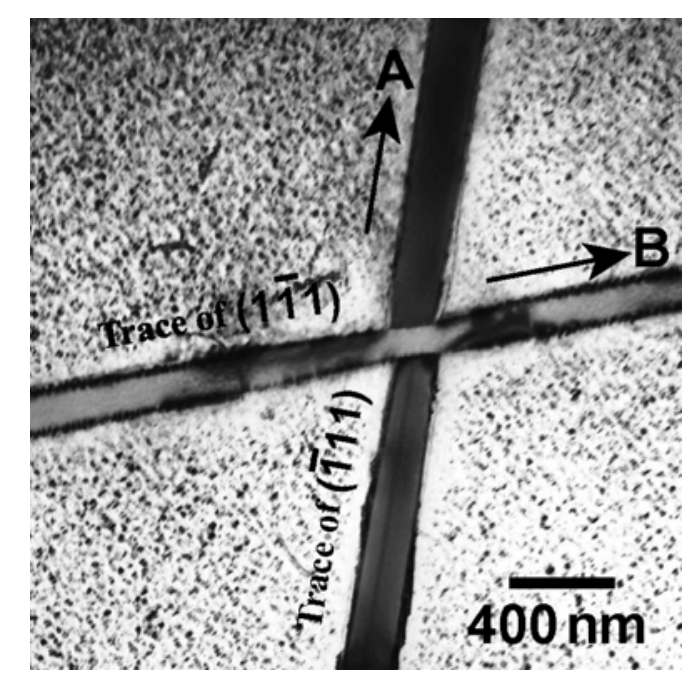

Figure 9. BF micrograph revealing one $\delta$ variant shearing another 
morphologies. However, SEM examination of deeply etched specimens unambiguously revealed that the $\delta$ particles had plate shaped morphology (Figure 7). This could be understood from the lattice parameter mismatches between the matrix and the $\delta$ phase. The lattice parameter of $\delta$ phase in Alloy 718 has been reported to be: ' $\mathbf{a}$ ' $=0.512 \mathrm{~nm}$, ' $^{\prime}$ ' $=0.423 \mathrm{~nm}$ and 'c' $=0.455 \mathrm{~nm}$ [9] and the ' $\mathbf{a}$ ' lattice parameter of the corresponding austenite matrix is $0.362 \mathrm{~nm}$. Along the ' $\mathbf{a}$ ' and ' $\mathbf{c}$ ' directions of the $\delta$ unit cell, the lattice misfit is very small, while in ' $\mathbf{b}$ ' direction it is high [9]. In order to reduce the overall strain energy, the $\delta$ particles assumed plate shaped morphology with the 'b' direction parallel to $\{111\}$ plane of the matrix that was consistent with the experimental observations.
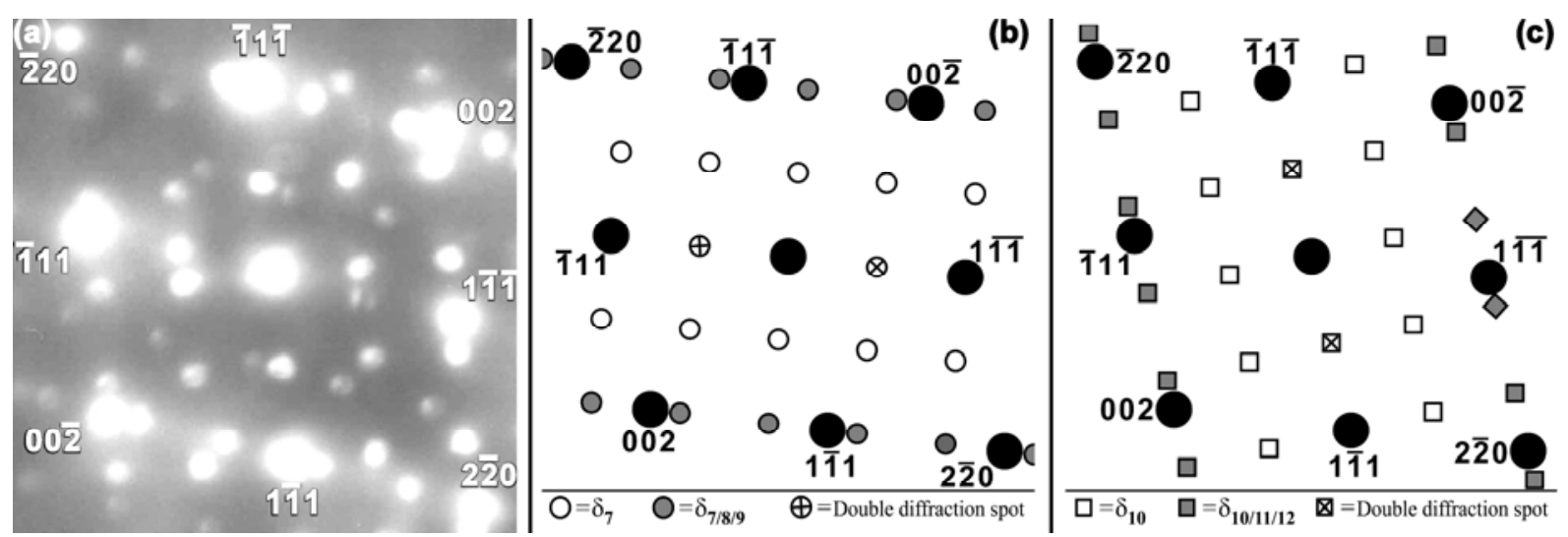

Figure 10. (a) [110] SAD taken from intersecting junction of $\delta$ precipitates shown in Fig 9. (b) and (c) are the key to SAD in (a) corresponding to $\delta$ plates B and A respectively.

The $\delta$ precipitation was noticed within the matrix, at grain boundaries and at coherent/incoherent segments of twin boundaries. The coherent boundary between the twinned and the untwinned region in the matrix, which was along $\{111\}$ planes, acted as the nucleation sites, since it was also the habit plane of the $\delta$ phase [9]. The nucleation of $\delta$ phase within the matrix could have occurred by any one of the following two processes. Crystallographically, the $\delta$ structure can be generated from a disordered fcc lattice by changing of stacking sequence from ...abcabc... type to ...abab... type [9] and subsequent ordering of minority atoms on close packed planes. Such a transformation would be initiated when the stacking fault energy of the material was low as in the present case. The other mechanism for $\delta$ formation is the generation of stacking faults within $\gamma^{\prime \prime}$ particles $[5,12]$ which were nothing but nuclei of $\delta$ phase [9]. Under favorable thermal conditions, these faults could grow easily into large sizes consuming the metastable $\gamma^{\prime \prime}$ particles. Evidence for such nucleation has been reported in the literature $[9,12]$. The aging temperature of $900{ }^{\circ} \mathrm{C}$ employed for DELTA samples is very close to the solvus temperature of the $\gamma^{\prime \prime}$ phase [23] and there is an overlap of $C$ curves corresponding to $\gamma^{\prime \prime}$ and $\delta$ phases. In the region of overlap, the metastable $\gamma^{\prime \prime}$ particles were found to precipitate first which transformed to $\delta$ phase on prolonged ageing [9]. In fact, earlier studies have shown that ageing at $900{ }^{\circ} \mathrm{C}$ for 20 hours resulted in profuse precipitation of $\gamma^{\prime \prime}$ particles with stacking faults within them. When the heat treatment period was increased to 100 hours, the microstructure consisted only of $\delta$ particles uniformly distributed within the matrix. Hence, it is reasonable to conclude that the intersecting $\delta$ structure had been generated by such a mechanism in the present study. The volume strain 


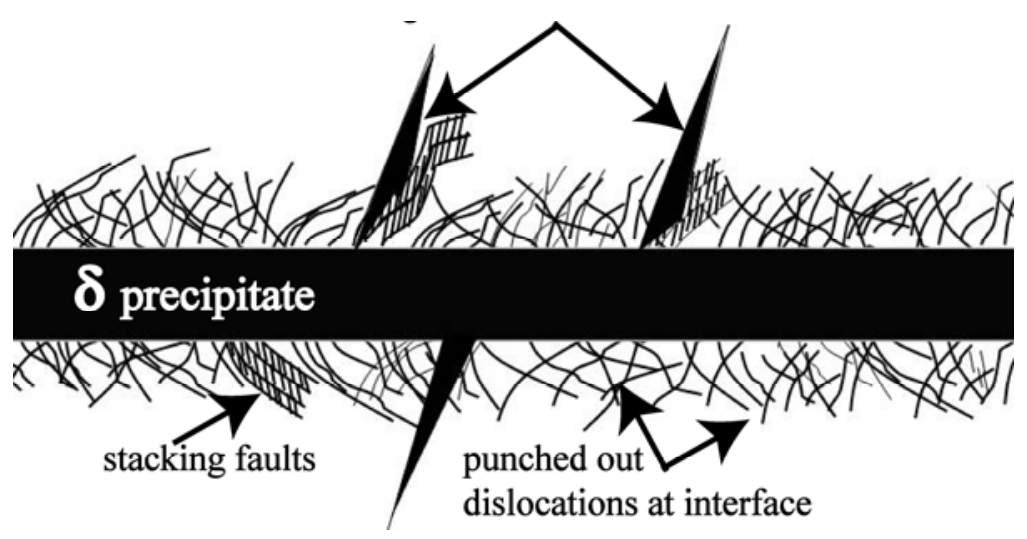

Figure 11. Schematic showing the nucleation of $\delta$ variant from the interfacial dislocations associated with another $\delta$ variant.

energy considerations indicated that the formation of $\delta$ particles within $\gamma^{\prime \prime}$ precipitates would be easier compared to homogeneous nucleation within the austenite at all temperatures below $\gamma^{\prime \prime}$ solvus [9]. It is, therefore, possible that in the present work $\delta$ precipitates have formed within the matrix predominantly by the latter mechanism.

In addition to the two mechanisms mentioned above, a new mechanism for the nucleation of the $\delta$ phase was noticed in the present study. As marked by dotted circles in Figure 8 , the $\delta$ particle corresponding to variant ' $A$ ' nucleated from variant ' $B$ ' of $\delta$ phase with another habit plane. The mechanism for the formation of such $\delta$ particles is proposed on the basis of dissociation of misfit dislocations at the interface or dislocations punched out near the interface to accommodate the growth strain. As is evident from Figure 8, plenty of misfit dislocations were generated at the $\delta$ matrix interface. Though it was difficult to characterize these interfacial dislocations owing to the overlapping diffraction contrast from $\gamma^{\prime \prime}$ precipitates in the matrix, it was reasonable to assume that majority of these dislocations are unit dislocations $(1 / 2<110>)$ of the matrix as $\{111\}$ is the interface plane. These perfect dislocations could dissociate into partials on a conjugate plane to locally reduce the strain around $\delta$ particles. Ordering of these faults, due to diffusion of niobium to it, would result in the formation of nuclei of another $\delta$ variant. Different habit plane variants of $\delta$ phase could be nucleated intragranularly within the matrix this way. A schematic diagram depicting the nucleation and growth of $\delta$ particles by a mechanism of this type is shown in Figure 11.

Often the $\delta$ plates belonging to two habit planes were found to intersect each other (Figures 6 to 8). A nucleation and growth of $\delta$ phase from stacking fault on conjugate plane discussed above could lead to such intersecting network. Different mechanisms leading to intersecting network of precipitates have been reported in the literature $[24,25]$. Three of them have been identified to be operating in the present case on the basis of microstructural observations.

Shear mechanism: In many areas, $\delta$ plates belonging to two habit plane variants were found to intersect producing a microscopic shear in the region of intersection in only one variant (Figure 9 ). From the reasonable matching of the estimated displacement with the theoretically calculated one (based on the assumption that $\delta$ particles are generated as a result of geometric stacking 
faults formation within $\gamma^{\prime \prime}$ particles, on every alternate layers and they grow by the propagation of these faults), one could argue that the intersecting morphology has occurred by one variant shearing the other during growth. Intersection of different variants of transformation product with plate shape morphology has been reported in the literature. Muraleedharan et al. [24] have reported that the shearing of one variant of ' $\mathrm{O}$ ' phase by another variant in $\mathrm{Ti}_{3} \mathrm{Al}$ system leads to the formation of original $\beta$ phase in the intersected volume. No such phase change at the junction was noticed in the present case (Figures 9 and 10). This has also been confirmed in an earlier investigation by the lighting up of the intersecting area when imaged in dark field, using superlattice reflections corresponding to one of the variant involved in the intersection [9]. It was possible that because of high temperature involved in the heat treatment, the region of intersection had transformed, in the present case, to one of the $\delta$ variant to reduce overall free energy.

Envelope mechanism: Detailed SEM examination of deeply etched $\delta$ specimens revealed that one variant appeared to be enveloped by another variant with a different habit plane during its growth (Figure 7). This observation suggested that when one $\delta$ variant meets another with different habit plane, during its growth, it could propagate in the same direction by wrapping around it. In fact, such a process has been put forward by Forwood and Gibson [25] to explain the propagation of twins in the same slip plane across many $\alpha_{2}$ lamellae in lamellar $\gamma$-TiAl alloys. According to the scenario suggested by them, twins could wrap around the termination of $\alpha_{2}$ lamellae and then invade the next $\gamma$ lamellae.

Strain transfer mechanism: Singh et al. [26] have proposed an alternate mechanism for the propagation of twins across $\alpha_{2}$ lamellae in two-phase TiAl alloys. According to their model, if a propagating twin hits an $\alpha_{2}$ lamellae, the elastic stresses are generated by it on the other side of $\alpha_{2}$ lamella. When this stress build-up is sufficient to nucleate twin there, then it would propagate without deforming the $\alpha_{2}$ lamellae at the point of intersection. Such a mechanism could be invoked in the present case as well. Since the $\delta$ plates are thin and their growth is very fast, high stresses are expected to be generated ahead of them. The $\delta$ variant intersection 3 , noticed in Figure 8 without any relative displacement, could have been generated by such a process. The observation of narrow width of variant $B$ at the junction for generation of intersecting $\delta$ particles microstructure indicated that high stress fields were generated ahead of them. This stress, on the right hand side of $\delta$ variant $A$ at junction 3, could be relieved by nucleation of the same variant $\mathrm{B}$ at left hand side.

\section{Conclusions}

The morphology of $\delta$ phase in the present work was found to be predominantly plate shaped. A new mechanism for nucleation of $\delta$ phase within the matrix has been proposed. The faults generated on the conjugate plane by dissociation of dislocations associated with $\delta$ particles could act as nuclei for formation of another $\delta$ variant with different habit plane. The possible mechanisms were identified as (i) one $\delta$ variant shearing another, (ii) a growing variant enveloping another variant with different habit plane lying in its path and (iii) the propagation of a $\delta$ variant across the obstructing variant by elastically mediated strain transfer. 


\section{Acknowledgements}

The authors wish to thank Dr. J.K. Chakravartty, Head, Mechanical Metallurgy Section and Dr. A.K. Suri, Director, Materials Group, B.A.R.C. for their constant support and encouragement.

\section{References}

1. E.A. Loria, eds., Superalloys 718, 625 and Various Derivatives, (Warrendale: TMS, The Minerals, Metals and Materials Society, 1989, 1991, 1994, 1999, 2001 and 2005).

2. H.L. Eiselstein, "Metallurgy of a columbium hardened nickel-chromium-iron alloy," ASTM Special Technical Publication, 369 (1965), 62-79.

3. D.F. Paulonis, J.M. Oblak, and D.S. Duvall, "Precipitation in nickel base alloy 718," Trans. ASM, 62 (1969), 611-622.

4. M.C. Chaturvedi, and Ya-fang Han, "Strengthening mechanisms in Inconel 718 superalloy," Met. Sci., 17 (1983), 145-149.

5. M. Sundararaman, P. Mukhopadhyay, and S. Banerjee, "Some aspects of the precipitation of metastable intermetallic phases in Inconel 718," Metall. Trans. A, 23, (1992), 2015-2028.

6. R. Cozar, and A. Pineau, "Morphology of $\gamma^{\prime}$ and $\gamma^{\prime \prime}$ precipitates and thermal stability of Inconel 718 type superalloys," Metall. Trans., 4 (1973), 47-59.

7. H.F. Merrick, Precipitation Processes in Solids, K.C. Russell and H.I. Aaronson eds., (Warrendale, PA: TMS-AIME, 1978) 161-201.

8. D.R. Muzyka, The Superalloys, C.T. Sims and W. C. Hagel, eds., (New York, NY: John Wiley, 1972) 113-43.

9. M. Sundararaman, P. Mukhopadhyay, and S. Banerjee, "Precipitation of the $\delta-\mathrm{Ni}_{3} \mathrm{Nb}$ phase in two nickel base superalloys," Metall. Trans. A, 19 (1988), 453-465.

10. E. Andrieu, N. Ang, R. Molinis, and A. Pineau, "Influence of compositional modifications on thermal stability of alloy 718," Superalloys 718, 625 and Various Derivatives, ed. E.A. Loria, (TMS, 1994), 695-710.

11. O.B. Armida, and J.F. Radavich, "A current T-T-T diagram for wrought alloy 718," Superalloy 718, 625 and Various Derivatives, ed. E.A. Loria, (TMS, 1991), 325-335.

12. I. Kirman, "Precipitation in the Fe-Ni-Cr-Nb system," J. Iron and Steel Inst., 207 (1969), 1612-18.

13. J.H Moll, G.N. Maniar, and D. R. Muzyka, "Heat treatment of 706 alloy for optimum $1200^{\circ}$ F stress-rupture properties," Metall. Trans., 2 (1971), 2153-2160.

14. Y. Desvallees, M. Bouzidi, F. Bois, and N. Beaude, "Delta phase in alloy 718: Mechanical properties and forging processes requirements," Superalloys 718, 625 and Various Derivatives, ed. E. A. Loria, (Warrendale, TMS, 1994), 281-291.

15. H.Y. Zhang, S. H. Zhang, M. Cheng and Z.X. Li, "Deformation characteristics of $\delta$ phase in the delta processed Inconel 718 alloy”, Materials Characterization, 61 (2010), 49-53. 
16. J. Dong, X. Xie, Z. Xu, S. Zhang, M, Chen and J. F. Radavich, "TEM study on microstructure behavior of Alloy718 after long time exposure at high temperatures", Superalloys 718, 625 and Various Derivatives, ed. E.A. Loria, (TMS, 1994), 649-658.

17. Y. Huang and T.G. Langdon, "The evolution of delta-phase in a superplastic Inconel 718 alloy," J. Mater. Sci., 42 (2007), 421-427.

18. S. Azadian, L. Wei, and R. Warren, "Delta phase precipitation in Inconel 718," Materials Characterization, 53 (2004), 7-16.

19. M. Sundararaman, "Microstructure of Alloy 718 and 625" (Ph.D thesis, Bombay University 1986).

20. D.R. Muzyka and G. N. Maniar, "Effects of solution treating temperature and microstructure on the properties of hot-rolled 718 alloy," Met. Engg. Quarterly, 9 (1969), 23-37.

21. V. Ramaswamy, P. Swann, and D R F West, "Observations on intermetallic compound and carbide precipitation in two commercial nicke-base superalloys," J. Less-Comm. Metals, 27 (1972), 17-26.

22. S.A Nalawade, "Deformation Behaviour of INCONEL 718 at Elevated Temperatures," (Ph.D thesis, Mumbai University 2009), 57-81.

23. C.T. Sims, N.S. Stoloff, and W.C. Hagel, Superalloys II (New York, NY: John Wiley \& Sons, 1987).

24. K. Muraleedharan, D. Banerjee, and S. Banerjee, "The $\alpha_{2}$ to $\mathrm{O}$ transformation in Ti-Nb-Al Alloys," Phil. Mag., 71 (1995), 1011-1036.

25. C.T. Forwood and M.A. Gibson, "Slip transfer of deformation twins in duplex $\gamma$-based Ti-Al alloys," Phil. Mag. A, 80 (2000), 2785-2811.

26. J.B. Singh, G. Molenat, M. Sundararaman, S. Banerjee, G. Saada, and P. Veyssiere, "In situ strain investigation of slip transfer across $\alpha_{2}$ lamellae at room temperature in lamellar TiAl alloy," Phil. Mag. Letters, 86 (2006), 47-60. 